\title{
Hospital Acquired Bloodstream Infection by Multi Drug Resistant Organisms-Alarming and Challenging Issue in the Intensive Care Units at Present Era
}

\author{
Barnini Banerjee $^{1}$ (D), Soumayan Mondal ${ }^{3}$, Vandana Kalwaje Eshwara ${ }^{1}$, \\ Chiranjay Mukhopadhyay ${ }^{1 *}$ (D) and Muralidhar Varma ${ }^{2}$ \\ ${ }^{1}$ Department of Microbiology, Kasturba Medical College, Manipal, Manipal Academy of Higher Education, \\ Manipal, Karnataka - 576 104, India. \\ ${ }^{2}$ Department of Medicine, Kasturba Medical College, Manipal, Manipal Academy of Higher Education, \\ Manipal, Karnataka - 576 104, India. \\ ${ }^{3}$ Kasturba Medical College, Manipal, Manipal Academy of Higher Education, Manipal, Karnataka - 576 104, \\ India.
}

\begin{abstract}
Hospital-acquired bloodstream infections (BSIs) cause high mortality in the intensive care units (ICUs) compared to wards. Furthermore, the isolation of multidrug resistant (MDR) organisms in ICUs add to the gravity of the condition making the treatment a bigger challenge. The present study was aimed to evaluate the prevalence, spread, and the possible MDR organisms contributing to ICU-acquired BSI \& the source of the secondary BSI in the ICUs. A prospective study was conducted in four ICU of tertiary teaching hospital over a period of six months. Patient that developed features of BSI within 48 hours after hospital admission were included in the present study Blood culture was performed by an automated BacT/ALERT ${ }^{\circledR}$ 3D system. The source of secondary BSI was identified by analysing culture results for the samples other than blood. These samples were taken within 48 hours of the patient's blood culture test being positive. A total of 50 patients (25\%) had an ICU acquired bloodstream infection. Out of 50 patients, $74 \%$ of them had bacterial etiology. Thirty one patients (62\%) among them developed multidrug resistant bacteraemia. Fifty six percent of Gram negative bacilli were multi drug resistant. The resistance to carbapenem was $42 \%$. The most common MDR Gram negative isolate was identified as Klebsiella pneumoniae $(n=12 ; 38.7 \%$,) and the most common source was pulmonary infection (26.7\% $(n=12) .42 \%$ of isolates in our study were carbapenem resistant suggesting the need for a proper antibiotic policy in the ICUs. Prolonged stay in the ICU with mechanical ventilation was the critical risk factor and ICU mortality was high (38\%).
\end{abstract}

Keywords: Intensive care unit, bacteremia, multidrug resistant, pulmonary infection

\footnotetext{
*Correspondence: chiranjay@gmail.com; + 918202933529
}

(Received: August 15, 2019; accepted: November 04, 2020)

Citation: Banerjee B, Mondal S, Eshwara VK, Mukhopadhyay C, Varma M. Hospital Acquired Bloodstream Infection by Multi Drug Resistant Organisms-Alarming and Challenging Issue in the Intensive Care Units at Present Era. J Pure Appl Microbiol. 2020;14(4):2595-2601. doi: 10.22207/JPAM.14.4.35

(C) The Author(s) 2020. Open Access. This article is distributed under the terms of the Creative Commons Attribution 4.0 International License which permits unrestricted use, sharing, distribution, and reproduction in any medium, provided you give appropriate credit to the original author(s) and the source, provide a link to the Creative Commons license, and indicate if changes were made. 


\section{INTRODUCTION}

Patients in intensive care units (ICUs) are at greater risk of developing nosocomial infections ${ }^{1}$. This problem is more in developing countries, largely due to lack of strict adoption of efficient infection control practices. The rate of nosocomial infections among ICU-admitted patients ranges from $11-60 \%{ }^{2}$. Bloodstream infections (BSIs) represent a major class of hospital acquired infection prevalent in ICU patients. It claims for approximately $20 \%$ of the ICU-acquired cases and affects $5 \%$ to $7 \%$ of hospital admissions ${ }^{3}$. The fatality rate can range from $35-50 \%$ further adding to the complexity 4 . It may present itself as primary BSI or a secondary BSI from an already existing source of infection in the patient ${ }^{5}$. BSIs acquired in the ICUs are due to drug-resistant hospital strains like Acinetobacter baumanii, $K$. pneumoniae, $P$. aeruginosa, $S$. aureus etc. These MDR pathogens increase the mortality rate, length of stay in the ICU, and generate substantial extra costs $^{6}$. It is necessary to know the risk factors, etiological agents, and source of secondary BSIs in the ICUs. These data can vary between institutions and ICUs within the hospitals. Hence each center should make its own therapeutic choice based on local surveillance data. Due to the lack of information in this area in the current study population, the study is being conducted to know the etiological agents of ICU acquired BSIs, their antibiotic susceptibility pattern, and the source of the pathogen to guide appropriate antimicrobial treatment.

\section{METHODS}

\section{Study design \& Population}

Prospective cross-sectional study was conducted in four ICUs under the control of a 2062-bedded tertiary-care teaching hospital of Karnataka. The study was conducted for six month duration from January to June 2018.

Adult patients who developed features of BSI 48 hours after admission to the ICUs were included. All patients with ICU admissions of $<=48$ hours and with existing BSI shifted from the ward or other hospital to the ICU were not included in the study.

Total of 200 patients' blood was sent for culture and antimicrobial susceptibility testing during this study period from four different ICUs after clinical suspicion of bacteremia. Among 200 patients, 97 of them fulfilled the inclusion criteria and were analysed further.

\section{Definitions}

Nosocomial infection was defined as an infection that occurred 48 hours after the patient's admission?.

ICU acquired BSI was defined as the patient having first positive blood culture two days after ICU admission without a prior positive blood culture with the same pathogen for at least 30 days.

A multidrug-resistant (MDR) microorganism has been defined as an isolate with resistance to at least one agent in three or more antimicrobial categories. Extensively drugresistant (XDR) microorganisms were defined as the organisms which exhibit susceptibility to only one or two antimicrobial categories ${ }^{8}$.

Secondary BSI was defined by the isolation of the same strain of microorganism from blood culture and a suspected source ${ }^{9}$. In the absence of a known source, ICU-BSI was defined as primary.

\section{Microbiology}

About 8-10 ml of blood was collected from the patients and then directly inoculated in the labeled BacT/ALERT ${ }^{\circledR}$ FA Plus media (bioMerieux, Inc, Durham, NC). The bottles were then sent to the microbiology laboratory to process in the BacT/ALERT ${ }^{\circledR}$ 3D system (bioMerieux, Inc, Durham, NC). This system works on the colorimetric principle. Specialized Liquid Emulsion Sensors (LES) at the bottom of each culture bottle visibly changes color from white to yellow when the $\mathrm{pH}$ changes due to the rise in $\mathrm{CO} 2$ as microorganisms produce it. BACT/ALERT ${ }^{\circledR} 3 \mathrm{D}$ instruments measure color changes. Positive flagged bottles contain microorganisms that were processed on $5 \%$ sheep blood and MacConkey agar and incubated at $37^{\circ} \mathrm{C}$ overnight. Simultaneously, Gram staining was performed directly from the bottle. The growth of microorganisms was identified by Matrix Assisted Laser Desorption lonization Time-of-Flight (MALDITOF) VITEK ${ }^{\circledR} \mathrm{MS}$, and antimicrobial susceptibility testing for the isolates was done by VITEK $^{\circledR} 2$ system (BioMerieux, Inc, Durham, NC).

\section{Data Collection and Analysis}

Data regarding patient demographics, admitting specialty, duration of stay in the 
ICU, presence of an underlying disease, details about antimicrobial therapy, and other relevant information was collected after taking the patient consent. The use of invasive devices and the number of days of their usage were also noted.

Culture results from other site-specific infections like lung infection, skin and soft tissue infection, urinary tract infection, intra-abdominal infection,, etc. were analysed within 48 hours of the positive blood culture to find out the source of secondary BSI. All positive blood cultures were then reviewed with reference to clinical records, imaging, and other laboratory data.

\section{Statistical analysis}

All the variables were presented as mean \pm standard deviation, median and interquartile range, frequency, and percentage as deemed appropriate. Univariate logistic regression analysis was done to determine the risk factors for acquiring bloodstream infection. Further, multivariate logistic regression analysis was carried out using the "Forward Wald" method to ascertain the independent predictors of acquiring BSI. All tests of significance were two sided with $p$-value $<0.05$ having statistical significance. Data were analysed using the Statistical Package for Social Sciences (SPSS) version 23.0 (Chicago, IL, U.S.A.) Unpaired t-test and Fischer's exact tests were used wherever applicable.

\section{RESULTS}

\section{Patient demographics}

A total of 1536 patients were admitted to the ICUs during the six months. Out of the 200 patients surveyed, 97 patients developed 106 nosocomial bacteremia episodes ( 88 patients with one episode and 9 patients with two episodes). Of these 97 patients, 50 (51.55\%) had an ICUacquired bloodstream infection, and the rest 47 patients $(49.45 \%)$ were diagnosed to have a non-

Table 1. Effect of patients related characteristics on the susceptibility of acquiring BSI

\begin{tabular}{|c|c|c|c|}
\hline Variables & $\begin{array}{l}\text { ICU-Acquired BSI } \\
\mathrm{N}=50(51.55 \%)\end{array}$ & $\begin{array}{l}\text { Non-ICU Acquired BSI } \\
\mathrm{N}=47(49.45 \%)\end{array}$ & $p$ Value \\
\hline Age (Mean \pm SD , Range) & $52.14 \pm 15.46(20-79)$ & $54.96 \pm 16.68 \quad(54-96)$ & $0.386 \#$ \\
\hline Male & $35(70)$ & $31(66)$ & \\
\hline Female & $15(30)$ & $16(34)$ & \\
\hline \multicolumn{4}{|l|}{$($ Mean $\pm S D)$} \\
\hline \multicolumn{4}{|l|}{$($ Mean \pm SD $)$} \\
\hline \multicolumn{4}{|l|}{ Underlying Co-morbid conditions ${ }^{\wedge}$ : } \\
\hline Diabetes & $19(38)$ & $12(27.7)$ & 0.281 \\
\hline Chronic Renal disease & $24(48)$ & $18(38.8)$ & 0.336 \\
\hline Cardiovascular disease & $12(24)$ & $4(8.5)$ & 0.048 \\
\hline Chronic liver disease & $15(30)$ & $14(29.8)$ & 0.982 \\
\hline Chronic lung disease & $19(38)$ & $13(27.7)$ & 0.281 \\
\hline Malignancy & $4(8)$ & $5(10.6)$ & 0.655 \\
\hline Neutropenia & $5(10)$ & $2(4.3)$ & 0.288 \\
\hline \multicolumn{4}{|l|}{ Exposure to invasive devices } \\
\hline Mechanical Ventilation & $36(72)$ & $22(46.8)$ & $0.035^{\wedge}$ \\
\hline \multicolumn{4}{|l|}{ No. of patients $n(\%)$} \\
\hline Duration of exposure (Mean \pm SD) & $8.12 \pm 5.36$ & $4.97 \pm 3.76$ & $0.024 \#$ \\
\hline Urinary Catheter & $26(52)$ & $14(29.8)$ & $0.091^{\wedge}$ \\
\hline \multicolumn{4}{|l|}{ No. of patients } \\
\hline Duration of exposure (Mean \pm SD) & $10.00 \pm 6.90$ & $6.60 \pm 5.41$ & $0.549 \#$ \\
\hline Central venous catheter(CVC) & $11(22)$ & $3(6.4)$ & $0.122^{\wedge}$ \\
\hline \multicolumn{4}{|l|}{ No. of patients } \\
\hline Duration of exposure (Mean \pm SD) & $10.44 \pm 6.62$ & $5.29 \pm 4.68$ & $0.227 \#$ \\
\hline
\end{tabular}

\#: Unpaired t-test^^: Fischer's exact test 
ICU acquired bloodstream infection. The incidence was 32.6 per 1000 admissions.

The mean (SD) age of the patients with ICU-acquired BSI was 52.14 years $( \pm 15.46)$ and $70 \%$ were male. The mean (SD) ICU stay was 12.78 ( \pm 11.31 SD) days. (Table-1)

Concomitant conditions and risk factors for bloodstream infection

The common underlying co-morbid conditions in patients with an ICU-acquired BSI were renal disease $(48 \%)$, diabetes $(38 \%)$, and pulmonary disease (38\%). There was also the presence of cardiovascular disease in 12 patients (24\%). (Table 1).
The results of exposure to invasive devices are shown in Table 1 . About $72 \%$ of patients were on mechanical ventilation during this course of ICU stay. The mean duration of a person being on mechanical ventilation was 8.12 days. Only $22 \%$ of the patients were exposed to a central catheter with a mean duration of 10.44 days.

\section{Microbiological profile of the ICU-BSI}

In patients with an ICU- BSI, 74\% were bacterial. Gram-negative bacteria (GNB) were the predominant organism isolated $(66.0 \%(n=33))$ followed by Gram-positive cocci and fungi (26\%

Table 2. Microbial profile of BSI

\begin{tabular}{lcc}
\hline Organisms Isolated & $\begin{array}{l}\text { ICU Acquired BSI } \\
\mathrm{N}(\%) \mathrm{N}=50\end{array}$ & $\begin{array}{c}\text { Non ICU Acquired BSI } \\
\mathrm{N}(\%) \mathrm{N}=47\end{array}$ \\
\hline Gram Positive Bacteria & $4(8)$ & $18(38.3)$ \\
Staphylococcus aureus & $1(2)$ & $15(31.9)$ \\
Enterococcus faecalis & $1(2)$ & $1(2.1)$ \\
Enterococcus faecium & $2(4)$ & $2(4.3)$ \\
Gram Negative Bacteria & $33(66)$ & $28(59.6)$ \\
Escherichia coli & $9(18)$ & $14(29.8)$ \\
Klebsiella pneumoniae & $15(30)$ & $6(12.8)$ \\
Enterobacter spp. & $2(4)$ & $3(6.4)$ \\
Serratia marcescens & $1(2)$ & - \\
Pseudomonas aeruginosa & $2(4)$ & $2(4.3)$ \\
Acinetobacter baumanni & $4(10)$ & $3(6.4)$ \\
Yeasts & $13(26)$ & $1(2.1)$ \\
\hline
\end{tabular}

Table 3. Antimicrobial susceptibility pattern of Gram-negative pathogens isolated from BSI

\begin{tabular}{lccccc}
\hline Antimicrobial drugs & $\begin{array}{c}\text { K. pneumoniae } \\
\mathrm{N}(\%)(\mathrm{N}=15)\end{array}$ & $\begin{array}{c}\text { E. coli } \\
\mathrm{N}(\%)(\mathrm{N}=9)\end{array}$ & $\begin{array}{c}\text { A. baumannii } \\
\mathrm{N}(\%)(\mathrm{N}=4)\end{array}$ & $\begin{array}{c}\text { Enterobacter } \\
\mathrm{N}(\%)(\mathrm{N}=2)\end{array}$ & $\begin{array}{c}\text { P. aerugonisa } \\
\mathrm{N}(\%)(\mathrm{N}=2)\end{array}$ \\
\hline Amikacin & $9 / 15(60)$ & $3 / 9(33.3)$ & - & $0 / 2(0)$ & $2 / 2(100)$ \\
Gentamicin & $8 / 15(53.3)$ & $7 / 9(77.8)$ & $3 / 4(75)$ & $0 / 2(0)$ & $2 / 2(100)$ \\
Ampicillin & $15 / 15(100)$ & $9 / 9(100)$ & - & $2 / 2(100)$ & - \\
Amoxicillin-Clavulinic & $12 / 15(80)$ & $7 / 9(77.8)$ & - & $2 / 2(100)$ & - \\
Acid & & & & $2 / 2(100)$ & - \\
Cefuroxime & $14 / 15(93.3)$ & $9 / 9(100)$ & - & $2 / 2(100)$ & - \\
Cefotaxime & $14 / 15(93.3)$ & $9 / 9(100)$ & - & - & $2 / 2(100)$ \\
Ceftazidime & - & - & $4 / 4(100)$ & $1 / 2(50)$ & $2 / 2(100)$ \\
Ciprofloxacin & $12 / 15(80)$ & $7 / 9(77.8)$ & $4 / 4(100)$ & $2 / 2(100)$ & $1 / 2(50)$ \\
Trimethoprim & $12 / 15(80)$ & $9 / 9(100)$ & $4 / 4(100)$ & & $2 / 2(50)$ \\
Sulphamethoxazole & & & & $0 / 2(0)$ & $2 / 2(100)$ \\
Cefepime & $11 / 15(73.3)$ & $7 / 9(77.8)$ & $4 / 4(100)$ & $0 / 2(0)$ & $2 / 2(100)$ \\
Cefoperazone-sulbactam & $11 / 15(73.3)$ & $4 / 9(44.4)$ & $3 / 4(75)$ & $0 / 2(0)$ & $2 / 2(100)$ \\
Imipenem & $11 / 15(73.3)$ & $4 / 9(44.4)$ & $4 / 4(100)$ & www.microbiologyjournal.org
\end{tabular}


Table 4. Statistics of acquiring BSI from different sources of infection

\begin{tabular}{lcc}
\hline Source & ICU- BSI & $\begin{array}{c}\text { Non ICU- } \\
\text { Aquired BSI }\end{array}$ \\
\hline Pulmonary Infection & $12(26.7)$ & $2(9.52)$ \\
Urinary Tract Infection & $6(13.3)$ & $7(33.33)$ \\
Surgical Site Infection & $2(4.4)$ & $2(9.52)$ \\
Digestive Tract Infection & $10(22.2)$ & $2(9.52)$ \\
Skin/Soft Tissue Infection & $1(2.2)$ & $0(0.00)$
\end{tabular}

$(n=13))$. Amongst the GNB, the most frequent organism isolated was Klebsiella spp. $(30 \%(n=15))$ followed by Escherichia coli $(18 \%(n=9))$ and Acinetobacter spp. $(8 \%(n=4))$. Enterococcus spp. was the major $(6 \%(n=3))$ isolates among Gram positive organisms. Among the fungi, Candida albicans, C. tropicalis, and C. parapsilosis were found in $8 \%(n=4)$ of the isolates.(Table 2 ).

\section{Antibiotic susceptibility pattern}

Total no. of MDR organisms isolated from BSI in the ICU was $62 \%(31 / 50)$. MDR Gramnegative pathogens dominated $(90.3 \%, 28 / 31)$ in ICU BSI. Frequently isolated MDR Gram-negative organisms were $K$. pneumoniae $(38.7 \%)$, E. coli (25.85\%), and A. baumanii complex (16.1\%). 91.7\% K. pneumoniae (11/12) , 75\% (3/4) Acinetobacter baumanii complex, and all Pseudomonas spp. were extensively drug resistant. $56 \%$ of (5/9) E. coli were $X D R$, and the remaining of them were ESBL producers. All the XDR strains were sensitive to colistin and aminoglycosides. ESBL producing GNBs were mostly sensitive to carbapenems $(88.9 \%$ $(n=9))$ followed by piperacillin-tazobactam $(85.7 \%$ $(n=7))$ and cefoperazone-sulbactam $(81.8 \%(n=9))$ (Table 3)

Among Gram-positive cocci, a single isolate of methicillin-resistant Staphylococcus aureus and two strains of Enterococcus faecium showing multi-drug resistance against penicillin, gentamycin, doxycycline, ciprofloxacin, and erythromycin.

\section{Source of bloodstream infection}

Out of 50 patients with ICU-acquired BSI, $38 \%$ had a primary bloodstream infection, and $62 \%$ of the BSI were secondary (Table 4). Amongst the secondary ICU-BSI, the most common source was pulmonary infection $(26.7 \%(n=12))$, followed by digestive tract infection $(22.2 \%(n=10))$.

\section{DISCUSSION}

The present study evaluated the etiological agents, antimicrobial susceptibility pattern, and source of secondary BSI acquired in intensive care of a tertiary care hospital. The incidence of ICU acquired BSI in our study was $6.4 \%$. A multicentre study (EPIC II) in ICUs conducted by Vincent et $\mathrm{al}^{10}$, showed that in ICU approximately $15 \%$ of patients had a BSI on the day of the study. Another study conducted by Sante et al. from 2009 to 2014 in a tertiary care hospital, documented $22 \% \mathrm{ICU}$ associated $\mathrm{BSI}{ }^{11}$.

The mean age of our cohort with an ICUacquired BSI was 52.14 years, and $70 \%$ was male. Our findings corroborate with a previous study in south India by Khan et al. which has also found that male patients at the age group of $40-50$ years had more frequency of ICU-acquired BSI ${ }^{12}$. It may be due to the presence of underlying comorbid conditions like diabetes, chronic renal disease, liver disease, hypertension in this age group of male patients. These conditions also made them visit the hospital several times and consequently increase the chance of exposure to several invasive devices. Zhang et al. also mentioned exposure to invasive devices as an important risk factor for bacteremia acquisition in this age group ${ }^{13}$.

Significant risk factors for ICU acquired BSIs are exposure to invasive devices, prolonged hospital stay, immunosuppression, etc ${ }^{3}$. Chronic renal disease (48\%), diabetes (38\%), chronic liver disease $(30 \%)$ were the main underlying co-morbid diseases noted in this study. A large number of patients also had the requirement of invasive devices like mechanical ventilation (72\%), urinary catheter (52\%), and central venous catheter (22\%). Aynur et al. analysed 65 patients with ICU-BSI and found chronic renal failure (CRF), diabetes mellitus (DM), mechanical ventilation and use of an arterial catheter, as significant risk factors ${ }^{14}$.

The ICU stay of the patients was estimated to be about $12.78 \pm 11.307$ days, and prolonged hospital stay significantly $(p=.007)$ attributed to the acquiring BSI in the ICUs. Adrie et al. analyzed 571 ICU-BSI occurring amongst 10,734 patients. They found a catheter-related infection, lung infection, and peritonitis were the major cause of secondary $\mathrm{BSI}^{9}$. In this study, ventilator associated pulmonary infection $(26.7 \%)$ was the most common source followed by abdominal 
infection (22.7\%) All the VAP causing strain led to $\mathrm{BSI}$ in the ICU were carbapenem resistant. Prolong ventilator days (8.12 \pm 5.36$)$ was a significant risk factor $(p=.024)$ of this. Hatem et al..$^{15}$ documented the ICU-BSI secondary to ventilator-acquired pneumonia in $25 \%$ of cases. VAP was reported to cause secondary bacteremia in $15-21 \%$ cases in another study ${ }^{3}$. Prolonged ventilation damage the host defense system particularly mucocilliary mechanism and helps the bacteria to invade the bloodstream.

In this study,Gram-negative bacteria predominated $(66 \%)$, which is consistent with the previous research ${ }^{5}$. Commonly isolated GNBs were Klebsiella pneumoniae, E. coli and Acinetobacter baumanni complex, which is similar to other ICU studies in southern India ${ }^{16,12}$. Klebsiella pneumoniae and Acinetobacter baumanni were the common organisms isolated in VAP associated bacteremia. Sante et al. also documented a similar findings in their study ${ }^{11}$. E.coli and Klebsiella predominated in the abdominal infection that led to the secondary case of BSI. Here, ESBLproducing Enterobacterales was challenging for clinicians, due to the resistance of the organisms to third generation cephalosporin. As consequence meropenem was the first choice of treatment and this may be due to high carbapenem resistance seen among isolates.

The epidemiology of infections in our ICUs has been primarily centered on the MDR organisms (62\%). High resistance to carbapenem (68\%)- non-fermenter GNBs (100\%) followed by Klebsiella pneumoniae(83\%) associated with the mortality of $38 \%$, which is similar to the study by Lijun Tian et al. ${ }^{17}$ Such a high percentage of MDR, especially carbapenem resistant, maybe due to inappropriate usage of antibiotics in the wrong dosage, failure of antimicrobial de-escalation, longer usage of broad spectrum antibiotics. This strongly suggests that meticulous infection control in ICUs may reduce ICU-related mortality in critically ill patients.

There were a few limitations to our study. Our study included only a few ICUs for a short period, which cannot be generalised for all types of ICUs globally or nationally. Detailed antimicrobial therapy data not included in this study which could have answered the high rate of MDR.

\section{CONCLUSION}

The present study was conducted in four medical ICUs of, a tertiary care teaching hospital of south Karnataka to guide the physicians on the most suitable antimicrobial algorithm to be followed. Multidrug resistant GNBs were the predominant group of organisms causing BSI in the ICUs,especially K. pneumoniae and A. baumannii. They showed resistance even to carbapenem. This study thus will help in effective formulation of antibiotic policy while emphasizing on the need to follow stringent infection control practices especially in the ICU.

VAP was the common source for secondary BSI in intensive care. Prolonged ICU stay and multiple co-morbidities significantly attributed to the risk of acquiring bloodstream infection. This indicates towards special care while dealing with patients on ventilator or other such devices especially with older patiensts or patients with reported history of co-morbidities in order to decrease the risk of acquiring a BSI. Also, early detection of acquired BSI in high-risk patients in order to start early therapeutic treatment is required.

\section{ACKNOWLEDGMENTS}

None.

\section{CONFLICT OF INTEREST}

The authors declare that there is no conflict of interest.

\section{AUTHORS' CONTRIBUTION}

BB was the guarantor of this research work and was involved in study design, literature search, and manuscript preparation; VKE \& CM was involved in the concept of the study, bacteriological evaluation and manuscript editing and review; SM was responsible for collection of samples, data analysis, statistical analysis, and interpretation of data. MV was involved in clinical analysis, manuscript editing and language editing. This manuscript has been read and approved by all the authors.

\section{FUNDING}

None. 


\section{DATA AVAILABILITY}

The data used to support the findings of this study are available from the corresponding author upon request.

\section{ETHICS STATEMENT}

An approval was obtained from the institutional ethics committee prior to commencement of the study.

\section{REFERENCES}

1. Barsanti MC, Woeltje KF. Infection prevention in the intensive care unit. Infect Dis Clin North Am. 2009;23:703-725. doi: 10.1016/j.idc.2009.04.012

2. Choudhuri AH, Chakravarty M, Uppal R. Epidemiology and characteristics of nosocomial infections in critically ill patients in a tertiary care Intensive Care Unit of Northern India. Saudi J Anaesth. 2017;11(4):402-407. doi: 10.4103/sja.SJA_230_17

3. Timsit J, Ruppe E, Barbier F, et al. Bloodstream infections in critically ill patients: an expert statement. Intensive Care Med. 2020;46:266-284. doi: 10.1007/ s00134-020-05950-6

4. Russotto V, Cortegiani A, Graziano G, et al. Bloodstream infections in intensive care unit patients: distribution and antibiotic resistance of bacteria. Infect Drug Resist. 2015;8:287-296. doi: 10.2147/IDR.S48810

5. Leal HF, Azevedo J, Silva GEO, et al. Bloodstream infections caused by multidrug-resistant gramnegative bacteria: epidemiological, clinical and microbiological features. BMC Infect Dis 2019; 19:609. doi: 10.1186/s12879-019-4265-z

6. Pittet D, Tarara D, Wenzel RP. Nosocomial bloodstream infection in critically ill patients: excess length of stay, extra costs, and attributable mortality. JAMA. 1994;271:1598-1601. doi: 10.1001/ jama.1994.03510440058033

7. Kappstein I, Schulgen G, Beyer U, et al. Prolongation of hospital stay and extra costs due to ventilatorassociated pneumonia in an intensive care unit. Eur J ClinMicrobiol Infect Dis. 1992;11:504-508. doi: 10.1007/BF01960804

8. Horan TC, Andrus M, Dudeck M. CDC/NHSN surveillance definition of health care-associated infection and criteria for specific types of infections in the acute care setting. Am J Infect Control. 2008;36:309-332. doi: 10.1016/j.ajic.2008.03.002

9. Adrie C, Garrouste-Orgeas M, Essaied WI, et al. Attributable mortality of ICU-acquired bloodstream infections: Impact of thesource, causative microorganism, resistance profile and antimicrobial therapy. Journal of Infection. 2017;74:131-141 doi: 10.1016/j. jinf.2016.11.001

10. Vincent JL, Rello J, Marshall J, et al., EPIC II Group of Investigators. International study of the prevalence and outcomes of infection in intensive care units. JAMA 2009;302(21):2323-2329. doi: 10.1001/ jama.2009.1754

11. Sante L, Aguirre-Jaime A, Miguel MA, Ramos MJ, Pedroso $Y$, Lecuona M. Epidemiological study of secondary bloodstream infections: The forgotten issue. J Infect Public Heal. 2019;12:37-42. doi: 10.1016/j. jiph.2018.08.011

12. Khan MF, Kundra P, Cherian A, Noyal J, Sistla S. Epidemiology of nosocomial infections in an intensive care unit at a tertiary care hospital in southern india: a retrospective study. Int J Infect Control. 2015;11(2). doi: 10.3396/IJIC.v11i2.016.15

13. Zhang $\mathrm{X}$, Tong $\mathrm{M}-\mathrm{M}$, Zhang $\mathrm{M}-\mathrm{Z}$, Zhu H.-P. "Risk factors of nosocomial bloodstream infections in surgical intensive care unit." Int J Clin Exp Med. 2015;8(9):16682-87.

14. Suner A, Karaoglan I, Mete AO, Namiduru M, Bosnak $\mathrm{V}$, Baydar I. Assessment of bloodstream infections and risk factors in an intensive care unit. Turk J Med Sci. 2015;45(6):1243-1250. doi: 10.3906/sag-1303-41

15. Kallel H, Houcke S, Resiere D, et al. Epidemiology and Prognosis of Intensive Care Unit-acquired Bloodstream Infection. Am J Trop Med Hyg. 2020;103(1):508-514. doi: 10.4269/ajtmh.19-0877

16. Saravu K, Prasad M, Eshwara VK, Mukhopadhyay C. Clinico-microbiological profile and outcomes of nosocomial sepsis in an Indian tertiary care hospital--a prospective cohort study. Pathog Glob Health. 2015;109(5):228-235. doi: 10.1179/2047773215Y.0000000026

17. Tian L, Tan R, Chen Y, et al. Epidemiology of Klebsiella pneumonia bloodstream infections in a teaching hospital: factors related to the carbapenem resistance and patient mortality. Antimicrob Resist Infect Control. 2016;5:48. doi: 10.1186/s13756-016-0145-0 